\title{
Combining data-driven neural networks of platelet signalling with large-scale ODE models of coagulation
}

\author{
M ANAND ${ }^{1, * \mathbb{D}}, \mathrm{M} Y \mathrm{LEE}^{2}$ and S L DIAMOND ${ }^{2}$ \\ ${ }^{1}$ Department of Chemical Engineering, Indian Institute of Technology Hyderabad, Kandi, Sangareddy 502285, \\ India \\ ${ }^{2}$ Department of Chemical and Biomolecular Engineering, University of Pennsylvania, Philadelphia, PA 19104, \\ USA \\ e-mail: anandm@iith.ac.in
}

MS received 20 September 2017; revised 28 February 2018; accepted 12 April 2018; published online 1 October 2018

\begin{abstract}
Platelet signalling during blood coagulation is conveniently modelled using data-driven neural networks since mechanistic Ordinary Differential Equation (ODE)-based models, while available, are unwieldy due to the large number of equations. On the other hand, the extra-cellular protease cascade of reactions that occur during blood coagulation are conveniently modelled using mechanistic ODE-based models. It is essential to integrate platelet signalling with the extra-cellular reaction cascade to get a representative model for in-vitro coagulation. In this paper, a neural-network-based platelet calcium calculator is combined with a large-scale ODE model for thrombin production. The combined model is used to test the effect of platelet modulators on thrombin production during in-vitro coagulation. The model predictions generate two hypotheses on relative importance of platelet modulators: they need to be compared to experimental data to confirm the same. The novelty of the study lies in the combination of existing approaches to study coagulation, and in capturing the role of platelet modulators on thrombin production: excessively large number of equations are avoided, which would be the case in a purely ODE-based approach.
\end{abstract}

Keywords. Coagulation; calcium calculator; neural network; ODEs; platelet modulation.

\section{Introduction}

Systems biology approaches have led to several models of blood coagulation in the last 15 years. Models may be classified as bottom-up (or mechanistic) or as top-down (or input-output correlation). Based on their treatment of platelets, mechanistic models may be further sub-classified as homogeneous, pseudo-homogeneous or heterogeneous. Platelets play a crucial role in the assembly of enzyme complexes that catalyse the formation of thrombin (fIIa), and their inclusion in mechanistic models has been a major challenge as reflected in the literature. For instance, the widely cited mechanistic model developed by Hockin et al [1] is only homogeneous, i.e., it considers only plasmabased zymogens while assuming that platelet-supplied phospholipid surfaces are available as required. The same is true for the model developed by Anand et al [2]. The model developed by Chatterjee et al in [3] is pseudo-homogeneous, and includes a coarse-grained description of platelet activation using a single variable. The recently developed Susree-Anand model in [4] is also pseudo-homogeneous, and includes platelets as a separate, yet soluble, reactant in

*For correspondence the model equations. The treatment of platelets is more detailed in the Kuharsky-Fogelson model [5] for extrinsic coagulation: their (mechanistic) model is heterogeneous, i.e., it includes the effect of platelet surface area (spatial spread) on coagulation. The mechanistic Bungay-Gentry model [6] for extrinsic coagulation is a heterogeneous model: it incorporates changes in the surface area of platelet membrane due to activation of phospholipid vesicles. However, in all these mechanistic models the complexity and interactions of platelet activation through signalling are at least neglected if not ignored.

The primary challenge in including the interactions involving activated platelets using a bottom-up mechanistic approach is the requirement of hundreds of reactions for completeness. A description of just adenosine di-phosphate (ADP)-mediated signaling of platelets, the Purvis-Diamond model [7], has 77 reactions! It will thus require hundreds more of such reactions to include collagen-mediated, thrombin-mediated and thromboxane (TXA)-mediated activation along with inactivation mediated by nitric oxide (NOX) and prostacyclin (prostaglandin $\mathrm{I}_{2}$ or $\mathrm{PGI}_{2}$ ). Thus, a pragmatic and computationally efficient approach to include platelet activation in a model for blood coagulation is to combine a top-down data-driven description of platelet 
activation with a mechanistic description of thrombin production. In this article, we do this by combining the data-driven platelet calcium calculator developed by Lee and Diamond [8] with the large-scale Ordinary Differential Equation (ODE) model developed by Chatterjee et al in [3].

The paper is organized as follows. We describe the method in section 2 . The results of simulations that mimic in-vitro clotting assays are given in section 3 , and discussed therein. The validated method, along with the limitations one may encounter while using it, and the hypotheses generated from the predictions are summarized in section 4.

\section{Method}

During initiation of simulations (time $t=0 \mathrm{~s}$ ), the platelet agonist/inhibitor concentrations corresponding to the case under study, and the physiologic concentrations of coagulation proteins in plasma (including thrombin) are obtained. For subsequent instants, the manner of obtaining these concentrations is given below.

\subsection{Platelet calcium calculator}

The concentration of intra-cellular calcium $\left.\left(\left[\mathrm{Ca}^{2+}\right]\right)\right)$ corresponding to time-varying input of multiple agonists (ADP, convulxin (CVX), thrombin, TXA, iloprost, NOX) for platelet activation is obtained using a platelet calcium calculator trained on 100 neural network scans [8]. The universal platelet calcium calculator has been developed such that it expanded the pair-wise agonist scan (PAS) method in [9] for use with exogenously added thrombin and platelet inhibitors: iloprost (analogue of platelet inhibitor $\mathrm{PGI}_{2}$ ) and NOX. The calcium calculator takes as input the instantaneous concentrations of ADP, CVX, thrombin, TXA, iloprost and NOX along with the history of intracellular calcium, and predicts a value for intra-cellular calcium released by activated platelets using a two-layer Artificial Neural Network (ANN).

During simulations, for a given time instant $t \mathrm{~s}$, the platelet calcium calculator is used to obtain the concentration of calcium $\left(\left[\mathrm{Ca}^{2+}\right]\right)$ at the next instant $(t+1 \mathrm{~s})$. Once the $\left[\mathrm{Ca}^{2+}\right]$ prediction is obtained, the $\left[\mathrm{Ca}^{2+}\right]$ in excess of baseline concentration of $100 \mathrm{nM}$ is integrated in time to generate an activation parameter $\xi$. This parameter is used to determine the instant when integrin expression occurs ( $\xi=\xi_{\text {crit }}=9 \mu \mathrm{M}$ s). The fraction of phospholipid expressed, for a given value of $\xi$, is obtained using a function fitted with data given in [10]: this fitting function is different from that used in [3]. The fraction of phospholipid thus obtained is equated to the extent of platelet activation $\epsilon$ at $t$ $+1 \mathrm{~s}$ : this is stored in an array that includes the history of $\epsilon$ values for all previous time instants up to $t \mathrm{~s}$. This $\epsilon$ array is fed to the ODE model. The ODE model then calculates coagulation protein concentrations at the next instant using the $\epsilon$ values up to $t+1 \mathrm{~s}$.

\subsection{ODE model}

We consider the generation of thrombin by addition of various concentrations of tissue factor (TF) in citrated plasma. The plasma is treated with various platelet modulators (ADP, TXA, CVX - a collagen analogue), iloprost, MRS (an ADP inhibitor)) and ASA (a TXA inhibitor) at the time of addition of TF. Coagulation occurs in static conditions in vitro. The enzymatic reactions are modelled by means of a system of 76 coupled ODEs. Each ODE is of the form given in Eq. (1):

$$
\frac{d\left[X_{i}\right]}{d t}=R_{i},
$$

where $\left[X_{i}\right]$ is the concentration of species $X_{i}$, and $R_{i}$ is the net rate of production (due to reaction/depletion) of species $X_{i}$. The individual expressions for each production term $R_{i}$ can be obtained from [3].

The presence of activated platelets is incorporated in the model by means of a scalar variable, $\epsilon$, which denotes the extent (between 0 and 1) of phospholipid expression by activated platelets. This scalar variable (which is governed by an ODE) is used to modify the kinetic constants that apply to the individual reactions in the following manner (see [3]):

$$
k_{\text {modified }}=k_{\text {original }}\left(\frac{\eta}{\epsilon}\right),
$$

where $\eta$ is an additional term used to adjust the original rate constant so as to fit the model predictions with experimental data. This manner of incorporating activated platelets is a coarse-grained description of the complicated signalling mechanisms that underlie the action of extracellular proteases on platelets.

The key difference, between the method in [3] and the method given in this paper, is in the manner of obtaining this term $\epsilon$. Whereas $\epsilon$ is obtained using an ODE in [3], we obtain it using $\left[\mathrm{Ca}^{2+}\right]$ predictions of a neural-networkbased platelet calcium calculator developed in [8] as explained in the previous section. At any given instant $t \mathrm{~s}$, the ODE model calculates the time variation of coagulation protein concentrations up to $t+1 \mathrm{~s}$ using the initial concentrations of coagulation proteins, and the $\epsilon$ array with values up to $t+1 \mathrm{~s}$.

A separate set of ODEs governs the platelet release of ADP and TXA according to the expression given in [11]: the released ADP and TXA contribute to more $\left[\mathrm{Ca}^{2+}\right]$ generation when input to the platelet calcium calculator because they trigger further activation of platelets. 


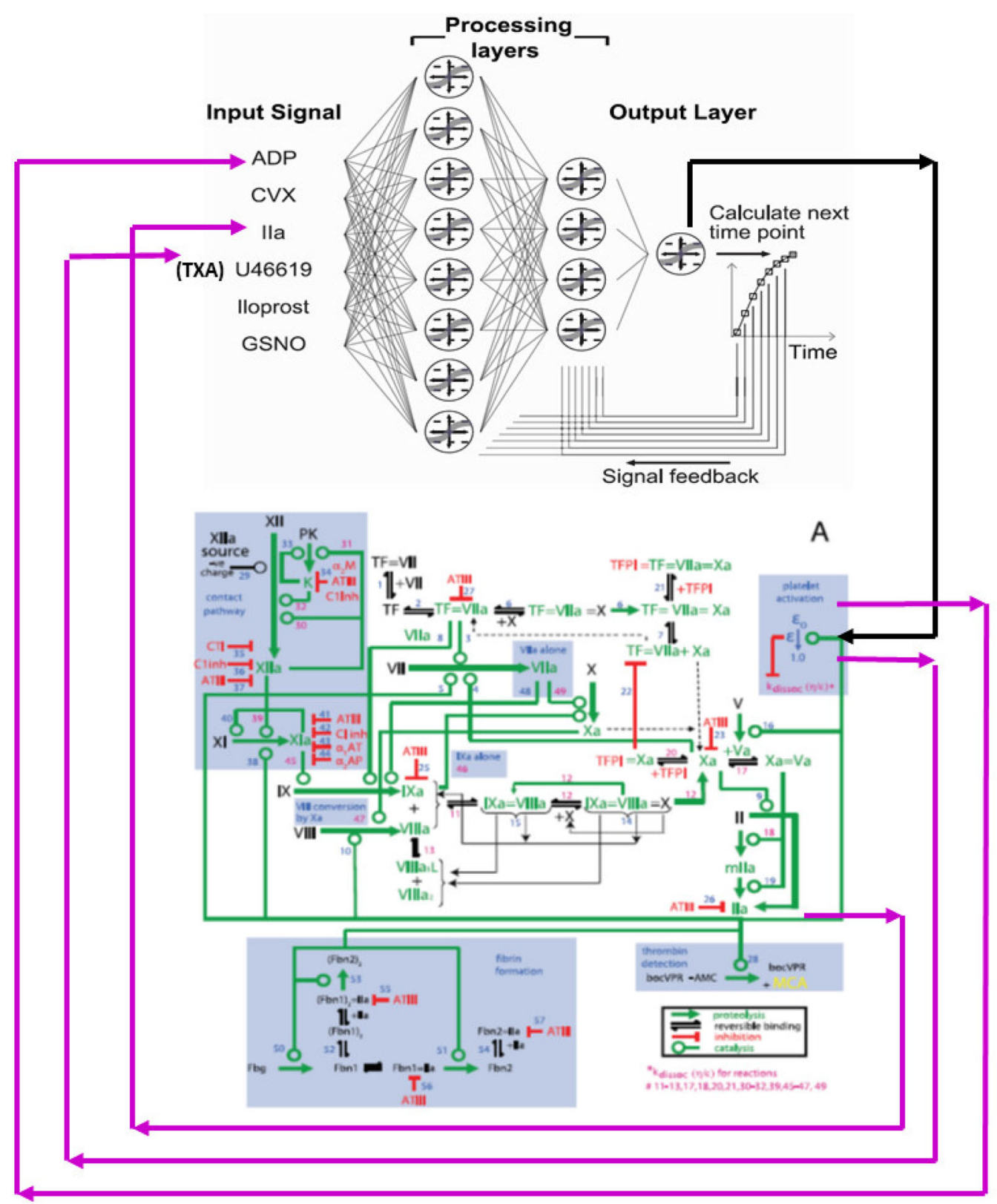

Figure 1. Combined ANN-ODE model for coagulation including platelet signalling; ANN schematic reproduced from [8], and ODE reaction network reproduced from [3] under Creative Commons Attribution (CC BY) license.

\subsection{Combining the platelet calcium calculator with the ODE model}

The solution procedure explained earlier indicates a decoupling of the solution for the calcium calculator and the ODE model. This decoupling is inevitable. The combined solution from the calcium calculator and the ODE model is made as simultaneous as possible by choosing the time step for time marching (in our case $1 \mathrm{~s}$ ) to be much smaller than the characteristic time of platelet signalling (30 s with CVX) or coagulation protein depletion $(\approx 450 \mathrm{~s}$ for peak thrombin with $[\mathrm{TF}]=5 \mathrm{pM}$ in $5 \times$ diluted whole blood as per simulations). Although we could explore smaller time instants, we have stayed content with the smallest time instant for which the platelet calcium calculator was developed. A perfectly simultaneous combination cannot be achieved because this would involve either using a full ODE model (which we have already noted will contain a prohibitive number of equations) or using a full ANN model (which will not have the mechanistic basis for the coagulation network that has been evolved through decades of research).

The agonist concentrations (ADP, thrombin, TXA) at $t \mathrm{~s}$, along with the history of agonist concentrations, are supplied to the platelet calcium calculator, which calculates the intracellular calcium concentration at $t+1 \mathrm{~s}$. The history of 


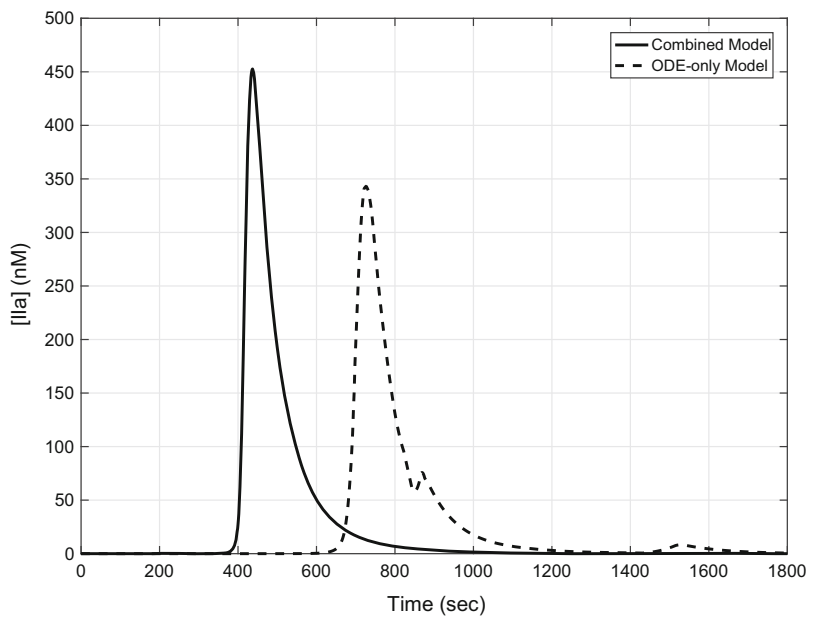

Figure 2. Thrombograms predicted by combined ANN-ODE model developed here and ODE-only model described in [3].

agonist concentrations (for all preceding times) is required by the platelet calcium calculator, and is obtained by concatenating the vector of agonist concentrations obtained at every instant. Note that only 3 agonist concentrations are changed at each instant; however, in principle, the process can be extended to all 6 agonists if ODEs governing the release of the other 3 agonists (CVX, iloprost, NOX) by platelets are also known/given.

The ODEs for thrombin generation are solved using a stiff solver (ode15s), accessing Gear's method using a specific options file, available in MATLAB R2015a. The ODEs use the percentage of activated platelets (up to $t+1 \mathrm{~s}$ ) obtained from the intra-cellular calcium concentration $\left(\left[\mathrm{Ca}^{2+}\right]\right)$ predicted by the platelet calcium calculator, with the initial condition set as the concentrations of the coagulation proteins at $0 \mathrm{~s}$.

The solution of the ODEs yields the concentration of thrombin (IIA) at the next instant $(t+1 \mathrm{~s})$. ADP and TXA are released by activated platelets, and their concentrations at $t+1 \mathrm{~s}$ are obtained by solving the ODEs given in [11] using a Runge-Kutta fourth order solver (ode45) available in MATLAB 2015a.

The cycle of obtaining the new $\left[\mathrm{Ca}^{2+}\right]$ as well as the ODE solution is then repeated at $t+1 \mathrm{~s}$, and continues for the specified amount of time. A schematic of the coupled ANN-ODE system is given in figure 1: this schematic includes the ANN (reproduced from [8]), which forms the backbone of the platelet calcium calculator, as well as the reaction network of the ODE model (reproduced from [3]).

\section{Results}

\subsection{Effect of platelet calcium calculator}

We first compare the thrombin concentration profiles (thrombograms) of the combined model developed here and the ODE-only model developed in [3]. Recall that the ODE-only model contains only a coarse-grained description of platelet signalling mechanisms. The thrombograms in figure 2 show that the combined model predicts much earlier onset of quantitative thrombin production $(\approx 450 \mathrm{~s}$ compared with $\approx 750 \mathrm{~s}$ ) as well as enhanced peak thrombin $(\approx 450 \mathrm{nM}$ compared with $\approx 350 \mathrm{nM}$ ) compared with the ODE-only model. This demonstrates the need for including the more accurate calcium calculator instead of the coarsegrained description for capturing the concentration of activated platelets $(\epsilon)$ during coagulation.

\subsection{Effect of platelet modulators}

We use the combined model to theoretically predict the effect of various platelet modulators by incubating citrated plasma (platelet count $=6 \times 10^{10} / \mathrm{ml}$ ) with the modulator at the instant of TF addition. The concentration of each modulator varied but was set at a value that would lead to detectable rise in intracellular [Ca]: the object in this exercise was to test the ability of the combined model to predict the response of the modulators rather than quantitatively assess the effect of each modulator. The incubating concentrations of ADP and U6619 (TXA analogue) are $10 E C_{50}{ }^{1}$ (i.e., 10 and $10 \mu \mathrm{M}$, respectively), concentrations of CVX and PGI are $E C_{50}$ (i.e., $2 \mathrm{nM}$ and $0.5 \mu \mathrm{M}$, respectively) and concentrations of MRS and ASA are $10 E C_{50}$ (i.e., 10 and $500 \mu \mathrm{M}$, respectively). Various concentrations of $\mathrm{TF}(0,0.1,1.0,5.0 \mathrm{pM})$ are introduced and $[\mathrm{TF}]$ and intracellular [Ca] are measured as time progresses; measurement is done for $30 \mathrm{~min}$. The predictions of the combined calcium calculator-ODE model are given in figures 3 and 4 for incubation with ADP, U6619 (TXA analogue), CVX, iloprost (analogue of $\mathrm{PGI}_{2}$ ), MRS and ASA. Further, the clot initiation time (defined as the time when $5 \%$ of BoC-VPR-MCA was cleaved) was also obtained for all these cases, and results are shown in figure 5. Of the six modulators mentioned, ADP, U6619 and CVX are activators, which contribute to increased release of intra-cellular calcium, whereas PGI, MRS and ASA are inhibitors, which prevent release of intra-cellular calcium; MRS inhibits ADP signalling, while ASA inhibits TXA signalling. We shall discuss only the results pertaining to $[\mathrm{TF}]=5 \mathrm{pM}$ because a further investigation of the theoretical results is not of value without corresponding experimental data, which were not obtained (this is a shortcoming of this study, and we record it in the subsection on Limitations).

\subsection{Discussion}

The results shown in figures 3-5 demonstrate the ability of the method to quantitatively predict the intra-cellular

$\overline{{ }^{1} E C_{50} \text { : effective concentration for half-maximum response. }}$ 


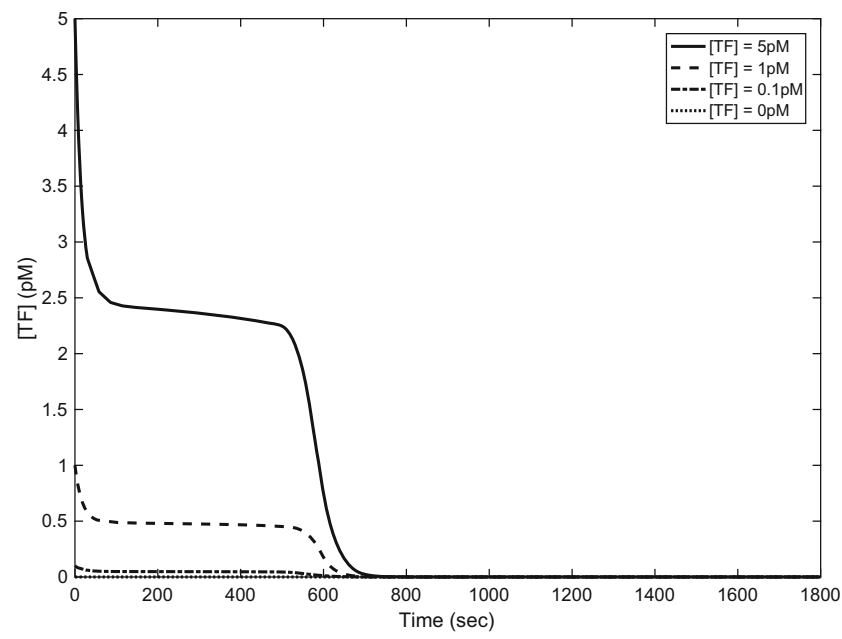

(a) $[\mathrm{TF}]$ vs $t(\mathrm{ADP})$

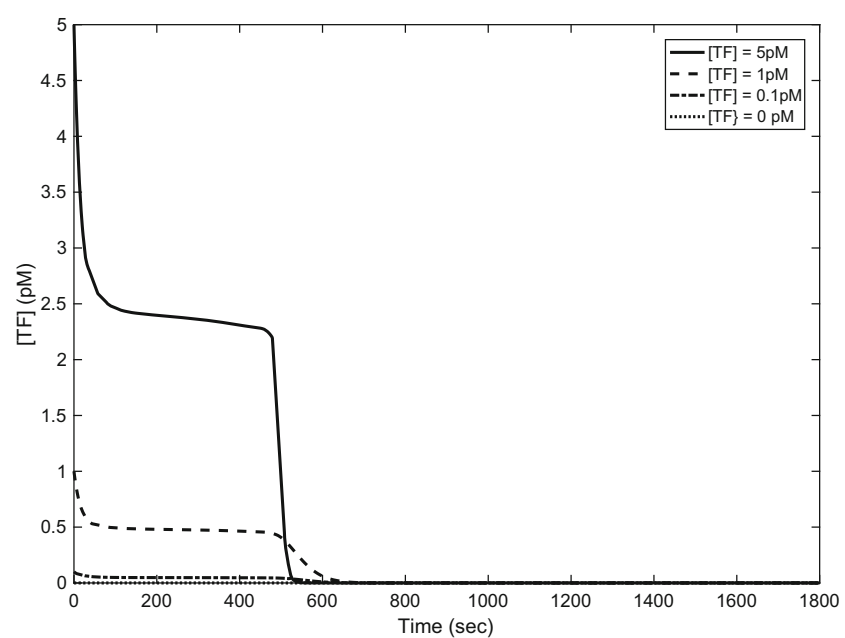

(c) $[\mathrm{TF}]$ vs $t(\mathrm{CVX})$

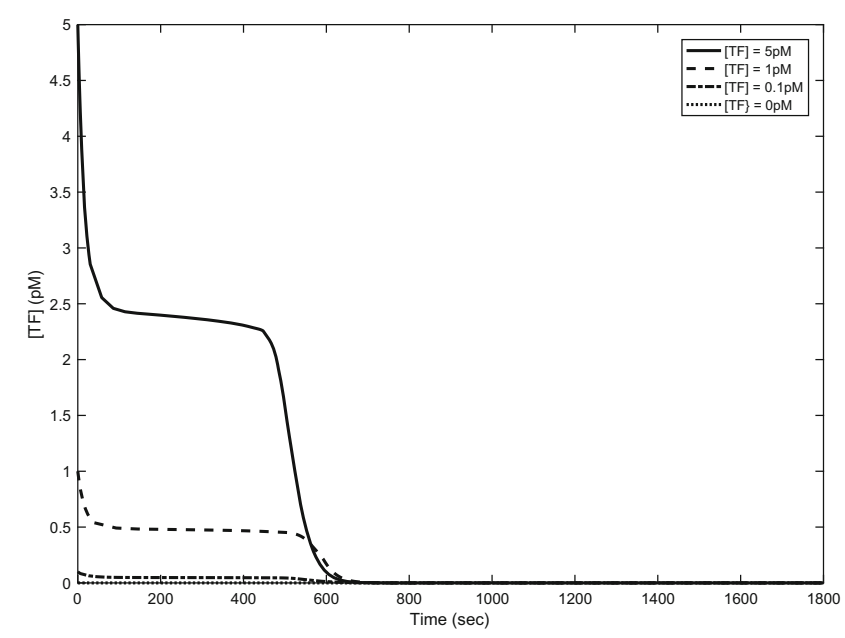

(e) $[\mathrm{TF}]$ vs $t$ (MRS)

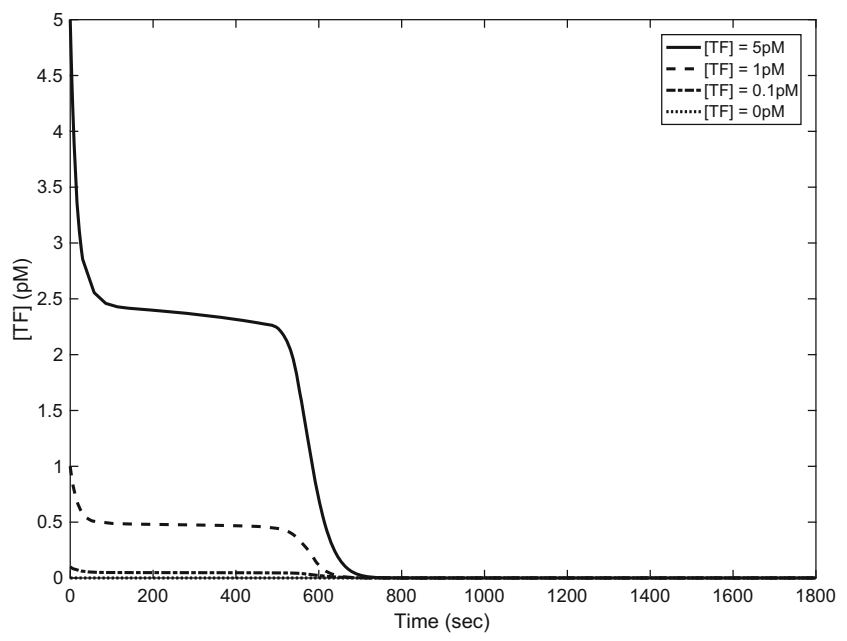

(b) $[\mathrm{TF}]$ vs $t$ (TXA)

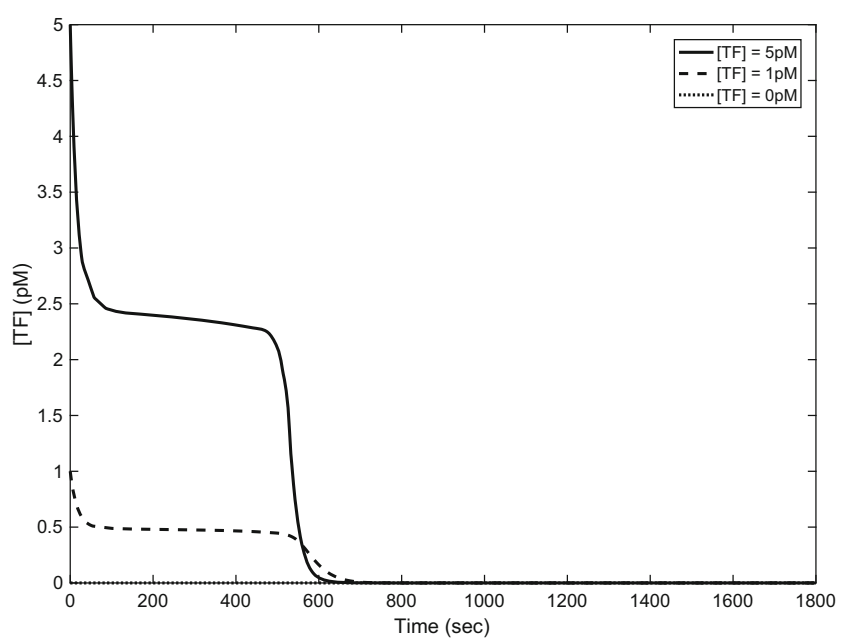

(d) $[\mathrm{TF}]$ vs $t$ (PGI)

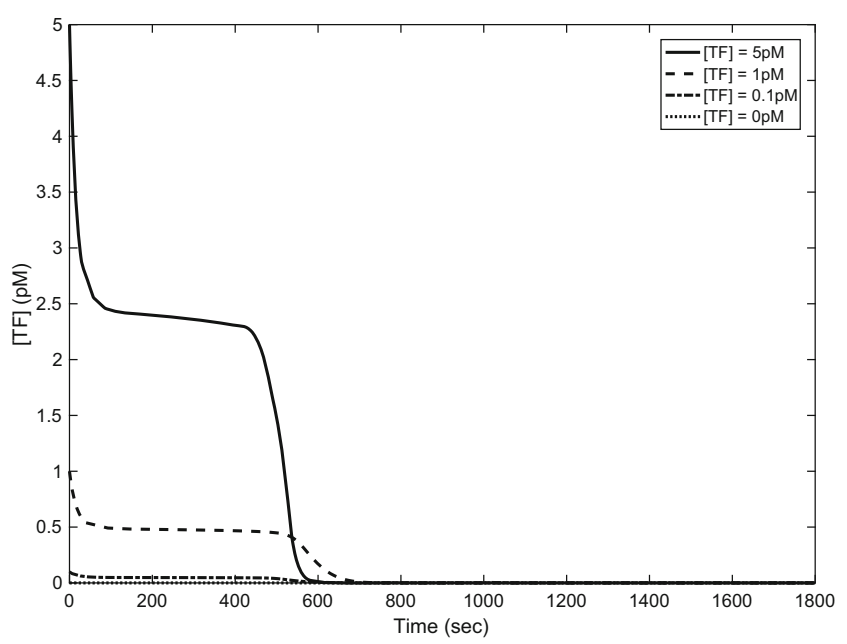

(f) $[\mathrm{TF}]$ vs $t$ (ASA)

Figure 3. Effect of platelet modulation on $[\mathrm{TF}]$ as predicted by combined ANN-ODE model. 


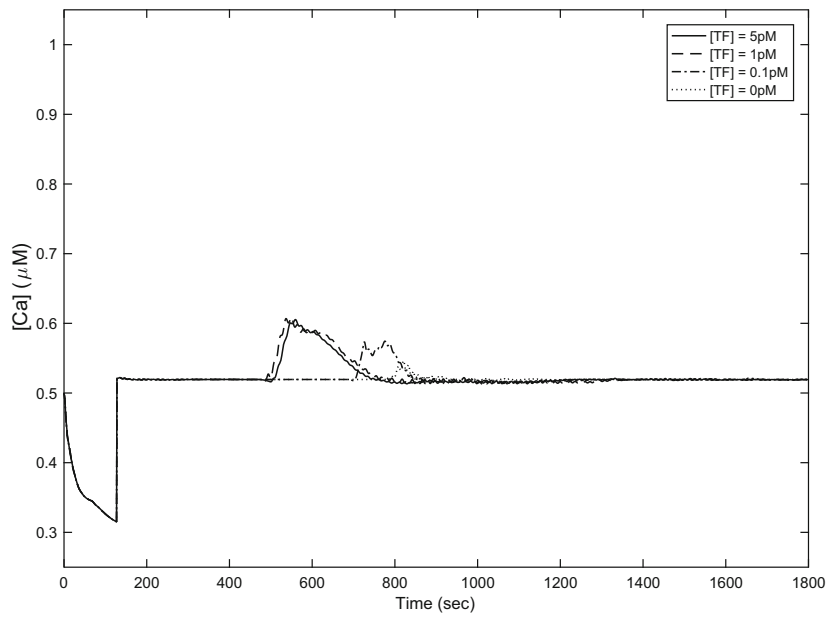

(a) $[\mathrm{Ca}]$ vs $t$ (ADP)

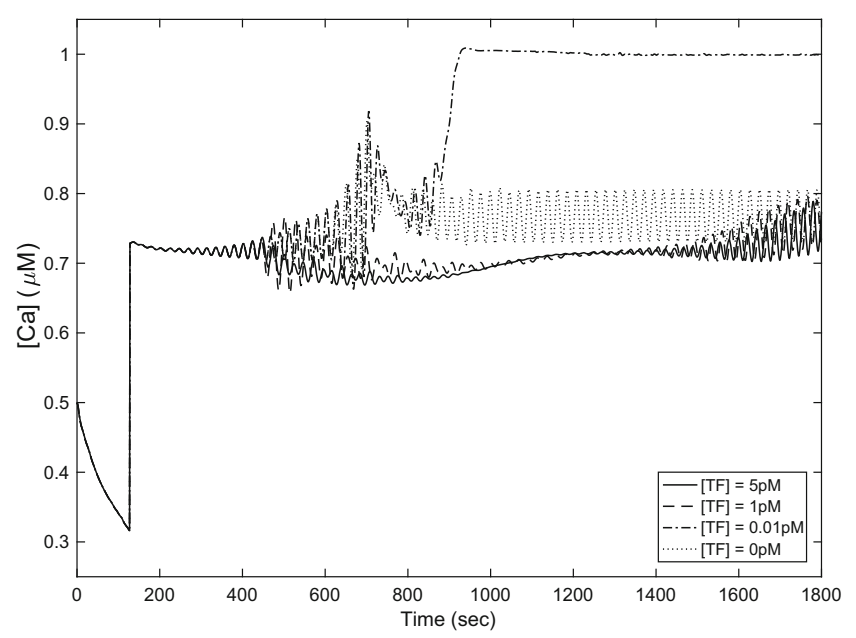

(c) $[\mathrm{Ca}]$ vs $t(\mathrm{CVX})$

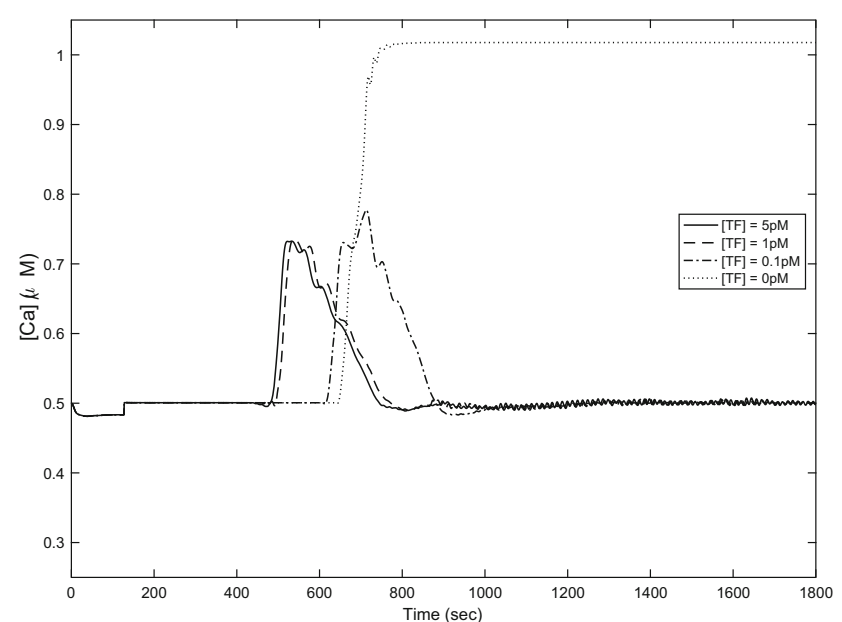

(e) $[\mathrm{Ca}]$ vs $t$ (MRS)

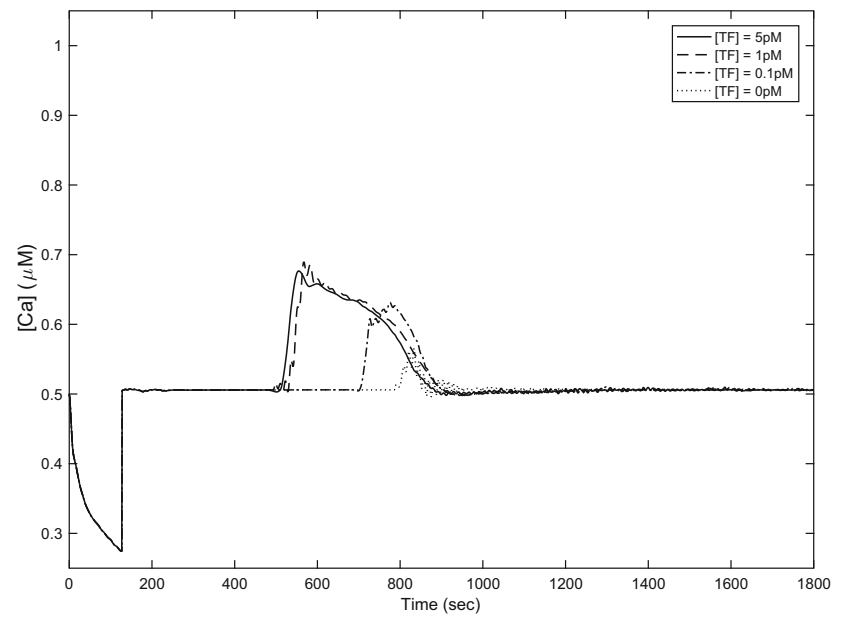

(b) $[\mathrm{Ca}]$ vs $t$ (TXA)

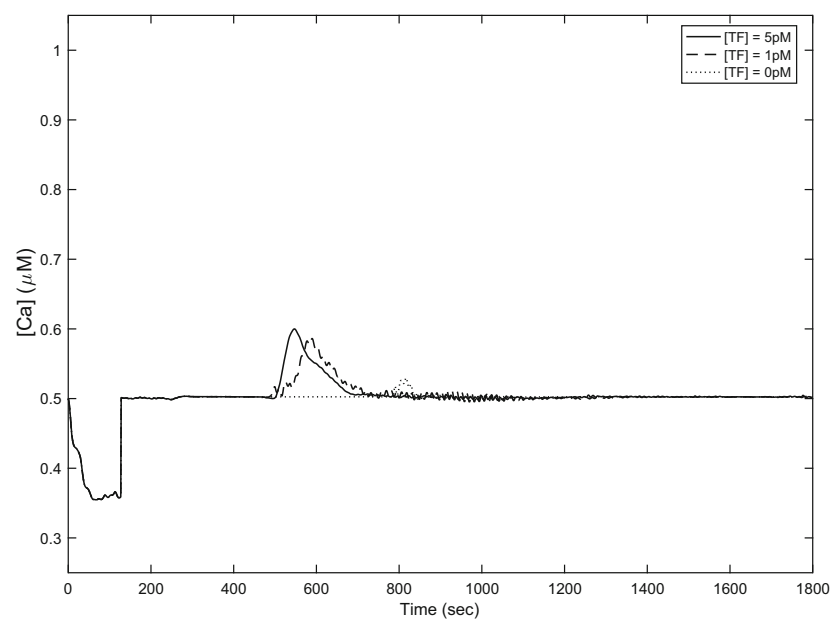

(d) $[\mathrm{Ca}]$ vs $t$ (PGI)

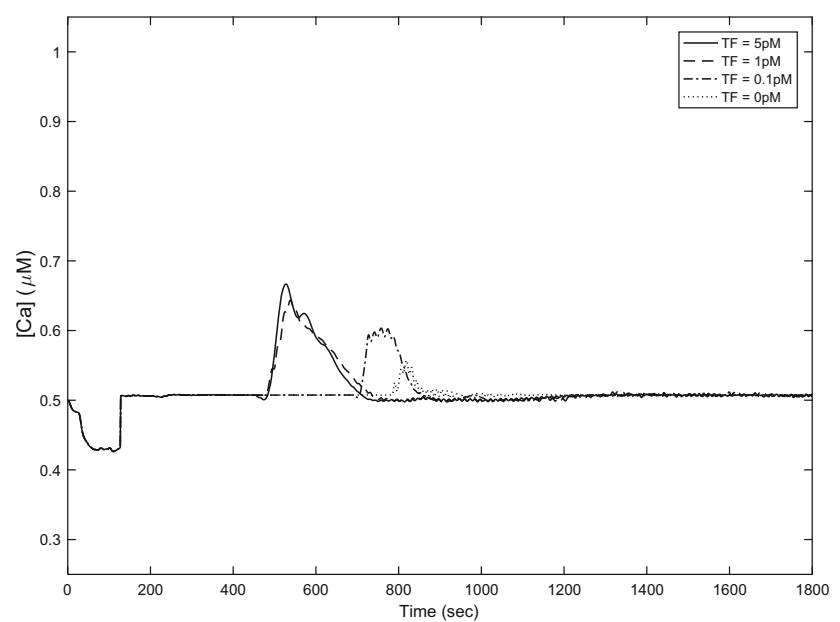

(f) $[\mathrm{Ca}]$ vs $t$ (ASA)

Figure 4. Effect of platelet modulation on $[\mathrm{Ca}]$ as predicted by combined ANN-ODE model. 


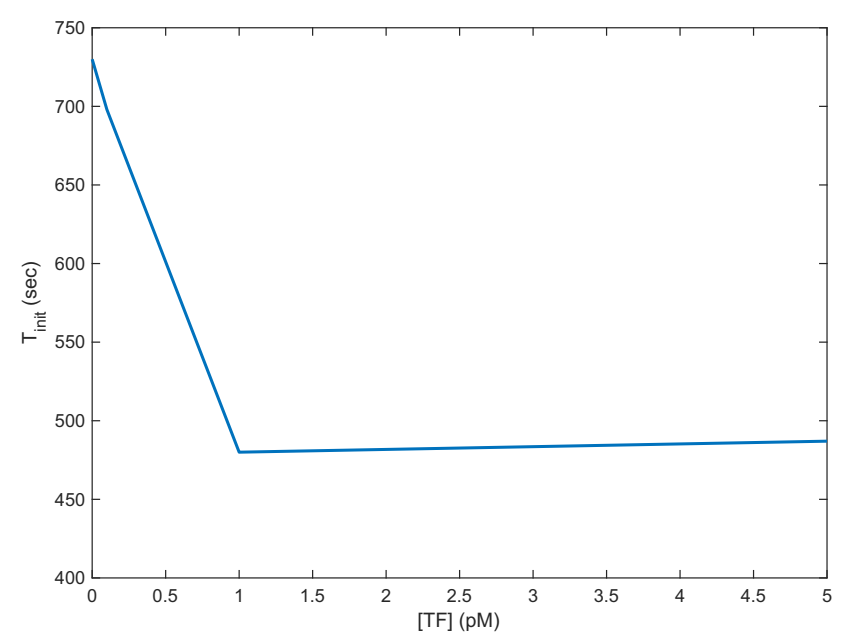

(a) $T_{\text {init }}$ vs $\mathrm{TF}(\mathrm{ADP})$

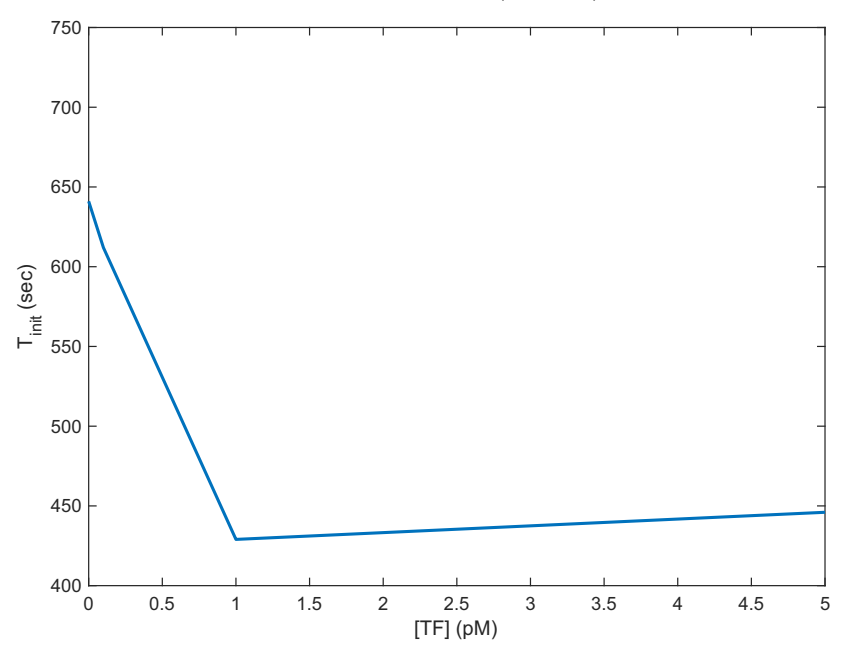

(c) $T_{\text {init }}$ vs TF (CVX)

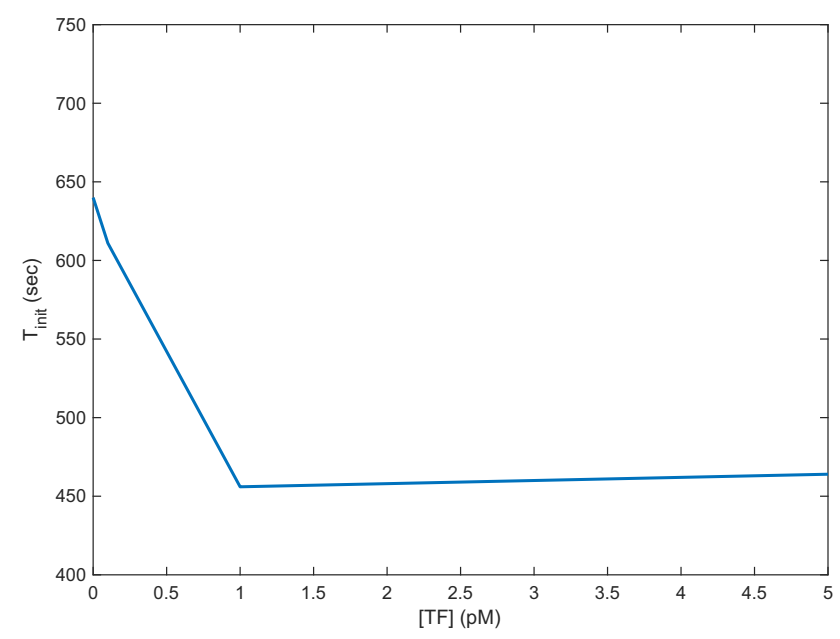

(e) $T_{\text {init }}$ vs TF (MRS)

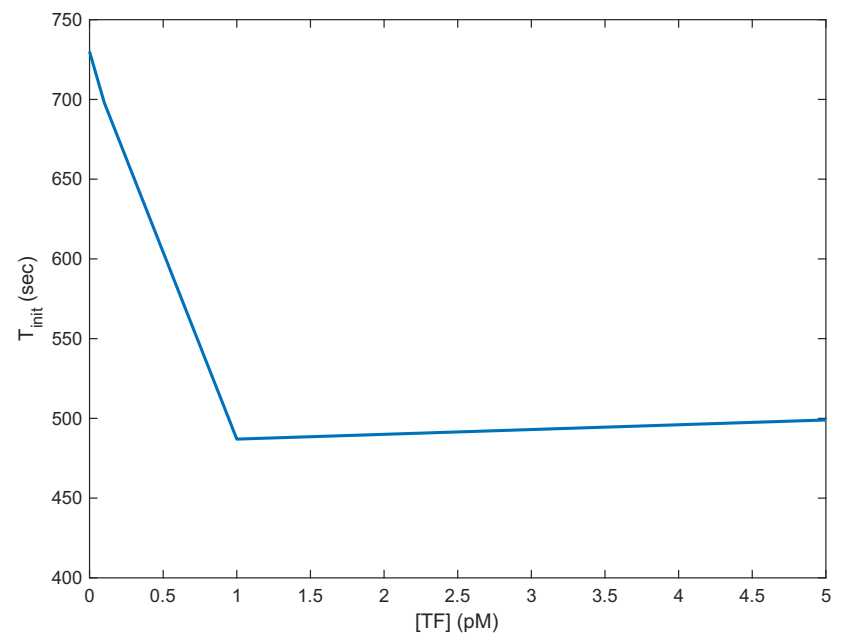

(b) $T_{\text {init }}$ vs TF (TXA)

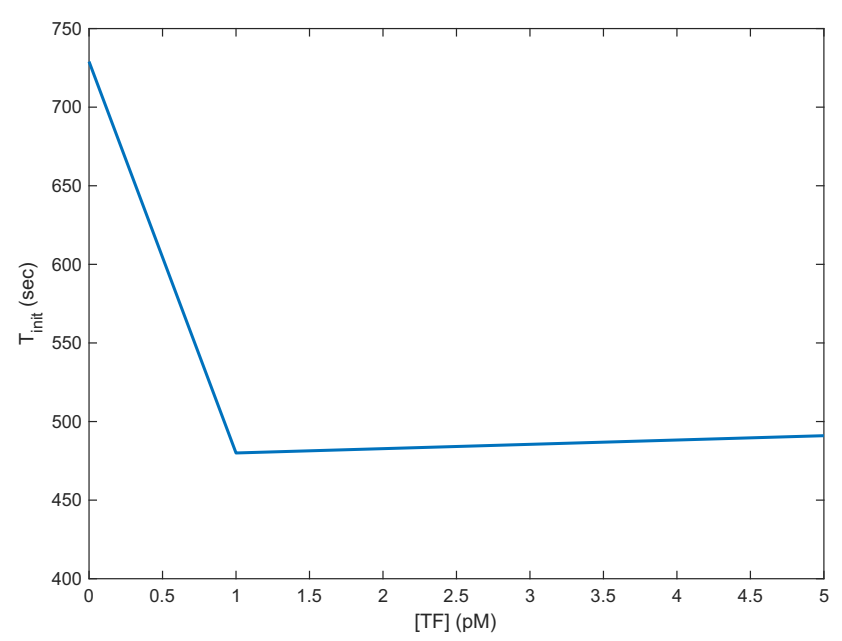

(d) $T_{\text {init }}$ vs TF (PGI)

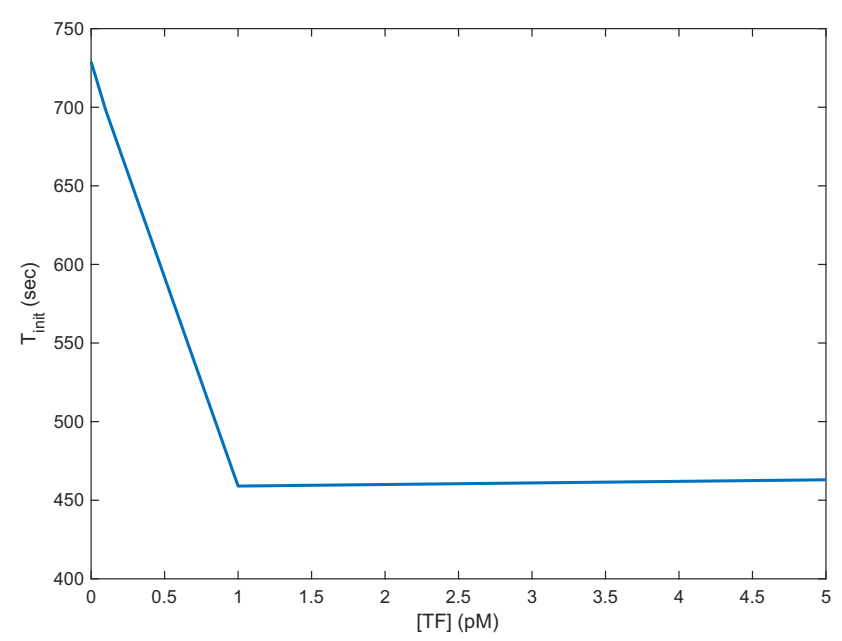

(f) $T_{\text {init }}$ vs TF (ASA)

Figure 5. Effect of platelet modulation on $T_{\text {init }}$ as predicted by combined ANN-ODE model. 
calcium and TF concentrations during coagulation of whole blood. Only qualitative hypothesis regarding the relative effect of each platelet modulator can be generated because the incubating concentrations of each modulator are different (recall that concentrations of ADP and U6619 (TXA analogue) are at $10 E C_{50}$, those of CVX and PGI are at $E C_{50}$, and those of MRS and ASA are at $10 E C_{50}(10$ and $500 \mu \mathrm{M}$, respectively) $)$. We will examine the hypotheses only for the case when $[\mathrm{TF}]=5 \mathrm{pM}$.

The results in figure 3 show that, for initial $[\mathrm{TF}]=5 \mathrm{pM}$, the concentration of TF drops to zero fastest when plasma is modulated with CVX (corresponding to collagen-induce signalling of platelets), and slowest when plasma is modulated with TXA. The increasing order (in terms of the platelet modulator) of time taken for [TF] to drop to zero is $\mathrm{CVX}<\mathrm{ASA}<\mathrm{PGI}<\mathrm{MRS}<\mathrm{ADP}<\mathrm{TXA}$. The noteworthy result here is that platelet modulation by CVX at $E C_{50}$ leads to faster consumption of TF than platelet modulation by either ADP at $10 E C_{50}$ or TXA at $10 E C_{50}$. This suggests that coagulation is much more sensitive to collagen-induced platelet signalling (whose analogue is CVX-driven platelet signalling) than to signalling by other activated platelets (which are responsible for ADP and TXA generation).

The results in figure 4 record that, at $[\mathrm{TF}]=5 \mathrm{pM}$, peak intra-cellular $\left[\mathrm{Ca}^{2+}\right]$ recorded is the least when plasma is incubated with PGI (which inhibits platelet activation) and the highest when incubated with CVX (which corresponds to collagen-induced platelet signalling via the GPVI receptor): this matches intuitive expectation. The increasing order (in terms of the platelet modulator) of peak intra-cellular $\left[\mathrm{Ca}^{2+}\right]$ recorded during TF-induced coagulation is $\mathrm{PGI}<\mathrm{ADP}<$ ASA $<$ TXA $<$ MRS $<$ CVX.

The results in figure 5 record that at $[\mathrm{TF}]=5 \mathrm{pM}$ the increasing order (in terms of platelet modulator used) of clot initiation time is given as follows: $\mathrm{CVX}<\mathrm{ASA}<\mathrm{MRS}$ $<$ ADP $<$ PGI $<$ TXA. The noteworthy result here is that at $[\mathrm{TF}]=5 \mathrm{pM}$ the initiation times for modulation by ASA and MRS (which are inhibitors of platelet activation) are actually less than those for modulation by ADP and TXA (which are accelerators of platelet activation) though not less than the initiation time for modulation by CVX. This suggests the hypothesis that coagulation at high [TF] is more sensitive to collagen-induced (CVX-driven) platelet signalling than to platelet signalling driven by ADP and TXA modulation.

\section{Conclusions}

We have combined a data-driven ANN-based platelet calcium calculator with a large-scale ODE model for thrombin generation, and obtained results of the combined model for modulation with six different platelet agonists.
Although the incubating concentrations of modulators do not facilitate quantitative assessment of the effect of each modulator, certain hypotheses may yet be deduced from the predictions of the combined model. The first prediction is that CVX, which initiates platelet signalling through the GPVI receptor in a manner similar to that of collagen, induces clot initiation and TF depletion much faster at $E C_{50}$ dose than even ADP at $10 E C_{50}$ : this suggests the hypothesis that collagen-induced platelet signalling dominates over platelet-driven platelet signalling during coagulation. The second prediction is that coagulation at high [TF] proceeds faster with ADP/TXA inhibition than with ADP/ TXA modulation though not faster than with CVX modulation. This suggests the second hypothesis, reinforcing the first hypothesis, that coagulation at high $[\mathrm{TF}]$ is more sensitive to collagen-induced platelet signalling than to platelet-driven platelet signalling.

\subsection{Limitations}

The neural network is a forcing function with noise that causes the solution of the ODE to oscillate due to stiffness: while these oscillations exist for $\left[\mathrm{Ca}^{2+}\right]$ predictions in all cases considered, the amplitude is too small to be noticed (typical range of $0.2-$ $0.5 \%$ ) except for the case of CVX modulation given in figure $4 \mathrm{c}$ (where it ranges between $5 \%$ and $10 \%$ ). This limitation needs to be noted when the method is adopted using the aforementioned solvers in MATLAB: it can be addressed by adopting a carefully selected numerical scheme for the system of stiff ODEs. Further, this study needs to be followed by one wherein the role of each modulator is assessed quantitatively by taking the same $E C_{50}$ equivalents for each modulator in theoretical predictions as well as validating experimental predictions (which also need to be obtained).

\section{Acknowledgements}

MA thanks IIT Hyderabad for funding the visit to University of Pennsylvania through the 2014 Summer Research Fellowship program.

Author Contributions MA coded the ANN-ODE model, performed simulations and wrote the article. MYL reviewed the results. SLD conceived and organized the study, and edited the article.

\section{References}

[1] Hockin M F, Jones K C, Everse S J and Mann K G 2002 A model for the stoichiometric regulation of blood coagulation. J. Biol. Chem. 277(21): 18322-18333

[2] Anand M, Rajagopal K and Rajagopal K R 2008 A model for the formation, growth, and lysis of clots in quiescent plasma: a comparison between the effects of antithrombin III deficiency and protein C deficiency. J. Theor. Biol. 253(4): 725-738 
[3] Chatterjee M S, Denney W S, Jing H and Diamond S L 2010 Systems biology of coagulation initiation: kinetics of thrombin generation in resting and activated human blood. PLoS Comput. Biol. 6(9): e1000950

[4] Susree M and Anand M 2017 A mathematical model for in vitro coagulation of blood: role of platelet count and inhibition. Sādhanā 42(3): 291-305

[5] Kuharsky A L and Fogelson A L 2001 Surface-mediated control of blood coagulation: the role of binding site densities and platelet deposition. Biophys. J. 80(3): 1050-1074

[6] Bungay S D, Gentry P A and Gentry R D 2003 A mathematical model of lipid-mediated thrombin generation. Math. Med. Biol. 20(1): 105-129

[7] Purvis J E, Chatterjee M S, Brass L F and Diamond S L 2008 A molecular signaling model of platelet phosphoinositide and calcium regulation during homeostasis and P2Y1 activation. Blood 112(10): 4069-4079

[8] Lee M Y and Diamond S L 2015 A human platelet calcium calculator trained by pairwise agonist scanning. PLoS Comput. Biol. 11(2): e1004118

[9] Chatterjee M S, Purvis J E, Brass L F and Diamond S L 2010 Pairwise agonist scanning predicts cellular signaling responses to combinatorial stimuli. Nat. Biotechnol. 28: 727-732

[10] Thiagarajan P and Tait J F 1990 Binding of annexin V/placental anticoagulant protein I to platelets: evidence for phosphatidylserine exposure in the procoagulant response of activated platelets. J. Biol. Chem. 265(29): 17420-17423

[11] Flamm M H, Colace T, Chatterjee M S, Jing H, Zhou S, Jaeger D, Brass L F, Sinno T and Diamond S L 2012 Blood 120(1): 190-198 